\title{
Processing of Integral-Skin Cellular Polymeric Composites in Rapid Rotational Foam Molding
}

\author{
R. Pop-ILIEV* \\ Faculty of Engineering and Applied Science, UOIT-University of Ontario Institute of Technology \\ Oshawa, Ontario, Canada L1H 7K4
}

\begin{abstract}
In order to survive the competition, the processing cycle time and the energy consumption of the rotational foam molding process must be reduced to a fraction of its current levels without compromising product quality. This paper introduces a novel extrusion-assisted rotational foam molding process for the manufacture of rotational moldings having adjacent, but clearly distinct, layers of an integrated solid (non-cellular) skin boundary layer encapsulating a cellular core structure, consisting of identical or compatible polyolefin grades, that achieves significant savings in processing cycle time duration and energy consumption. It introduces non-chilled extruded foam as a foamed core-forming material, in real time, directly into a uni-axially rotating hot mold through a dedicated mold "injection" port onto the already formed un-foamed soft skin layer.
\end{abstract}

PACS: 47.32.Ef, 81.20.Hy, 82.70.Rr, 47.57.Bc, 83.80.Iz, 83.80.Sg, 61.25.hk, 47.85.md, 81.05.Qk, 83.80.-k

\section{Introduction}

The rotational molding process inherently suffers from very lengthy and energy-intensive processing cycles the duration of which is extended even further if producing integral-skin foamed moldings. This delay, attributed to the insulative effects of the thereby formed cellular structure, especially during the mold cooling segment. When combined with the thermal gradients across the mold, it directly governs the efficiency of the processing control in terms of aggravating the simultaneous retention of finecelled foam morphologies while maintaining a uniform thickness of the solid skin. Thus, the greatest unresolved disadvantage of contemporary rotational molding technologies is the lengthy cycle time and the lack of real-time or "quick enough" means of heat transfer process control.

\section{Background}

Essentially, the manufacture of rotational moldings with a distinct non-foamed outer skin that encapsulates entirely a foamed core or layer requires both nonfoamable and foamable plastic resins to be charged into the mold within an identical rotational molding cycle. Recently developed, single-charge concepts are based on charging the mold with a mixture of predetermined quantities of both non-foamable and foamable resins simultaneously at the outset of the cycle, so that the use of drop boxes or plastic bags becomes unnecessary. Although the single-charge processing concept is beneficial for improving the efficacy of the molding process and the structural

* e-mail: remon.pop-iliev@uoit.ca homogeneity of the moldings, it certainly suffers from inherently aggravating the fulfillment of crucial processing goals such as: (i) the execution of the adhesion of the non-foamable thermoplastic resin to the internal surface of the mold that should always take place prior to the thermal activation of the foaming resin (thereby avoiding skin protrusions), and (ii) obtaining a solid skin layer with a uniform thickness [1-3, 6-11].

The control of the cell size of rotationally foam molded foams formed on the base of a chemical blowing agent might be often aggravated by some inherent limitations that are unique to the rotational molding process. For example, lengthy processing cycles often result in coarsercelled final cellular structures being yielded. In addition, inherently, the polymer close to the internal mold surface continues to be heated even after its foaming is completed, simply because the interior polymer has yet to reach the decomposition temperature of the blowing agent. Even when cooling is applied to the mold, the recrystallization temperature in the melt is reached after several minutes, during which time bubble coalescence and/or collapse sets out and accelerates [12].

It is also inherent to the rotomolding process that, as the melt front progresses, the air pockets that have been entrapped inside the melt eventually become bubbles that will be subjected to diffusion controlled shrinkage and eventual disappearance [13]. At a high melt temperature, the air in the bubbles begins to dissolve into the polymer. Since oxygen has about twice the solubility of nitrogen in polyethylene, at high temperatures, the oxygen is further depleted by direct oxidation reactions with polyethylene, which depletion reduces the bubble diameter. Surface tension laws dictate that the pressure inside the bubble has to increase as the diameter decreases, 
which forces nitrogen to dissolve in the polymer thereby further reducing its diameter. Normally, this occurrence repeats until the bubble disappears [14].

A fundamental research of the lifespan of CBA-blown bubbles in non-pressurized non-isothermal polymer melts using hot-stage optical microscopy and digital imaging indicated that the lifespan of fine-celled bubbles is significantly shorter than the inherently lengthy heating portion of the rotational molding process, so that fine-celled bubbles seldom reach the solidification stage of the cycle, which implies that only coarser-celled bubbles live long enough to participate in the final cellular structure $[15,19]$. One of the major progresses of this research includes the development of a two-step oven temperature profile that prevents the foamable resins invading the solid skin layer and ensures that skin formation always completes prior to the activation of the foamable resin. It was based on the fundamental study of the adherence behavior of powders and foamable pellets to a high-temperature rotating mold wall and a fundamental study of the rotofoamablility of polyolefin resins using both dry blending and melt compounding based methods including the characterization of their respective rheological and thermal properties. This study clarified why in rotationally foam molded cellular structures based on the use of a CBA, a fine-celled morphology has been closely approached, but it has not been actually achieved yet. Thus, it was clearly indicated that it would be very difficult to generate the preferred fine-celled kind of bubbles (cell size $<100$ micron and cell density $>10$ million cells $/ \mathrm{ccm}$ ) in rotational foam molding unless the duration of the processing cycle would be dramatically reduced, or else [7, 15-20]. The presently presented research is intended to propose a remedial solution.

\section{Rapid rotational foam molding}

A custom-build experimental setup dedicated to Rapid Rotational Foam Molding is schematically presented in Fig. 1, whereas its processing concept can be summarized into four unique steps that are depicted in Fig. 2.

Step 1: At the inception of the process, the mold is manually charged with a predetermined amount of nonfoamable plastic resin in powder form, which will be used to create the part's solid skin.

Step 2: The arm and the mold assembly are inserted into the oven by means of a carriage and translational mechanism. The mold rotates in a biaxial manner within the oven, utilizing the arm. This step is accomplished at a material-dependent elevated temperature for an accordingly set period of time to accomplish the creation of the skin. Simultaneously, the extruder is charged with predetermined amounts of dry blended polymer resin with CBA, suitable for achieving polymeric foam that exhibits a desired volume expansion ratio (VER) at a set melt temperature.

Step 3: The arm and mold assembly are then translated towards the extruder, with the arm rotation

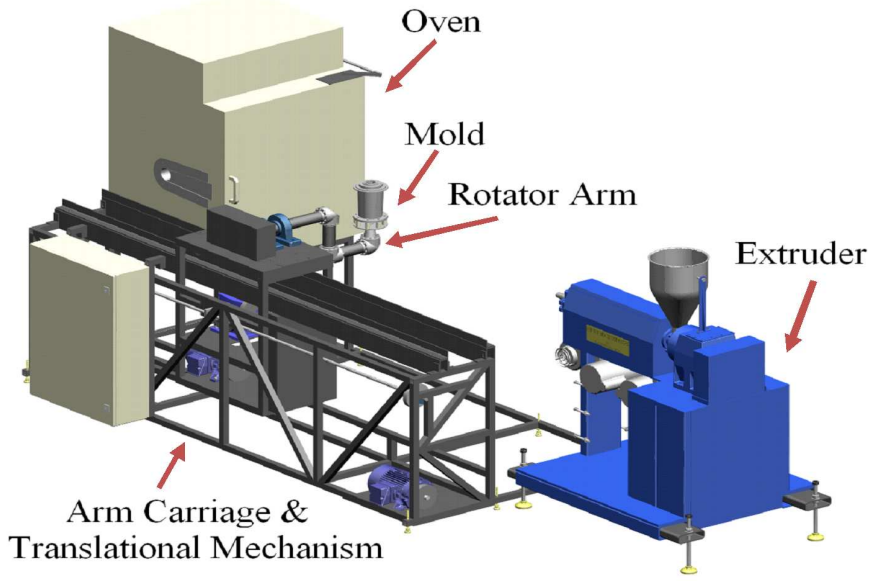

Fig. 1. Experimental setup design.

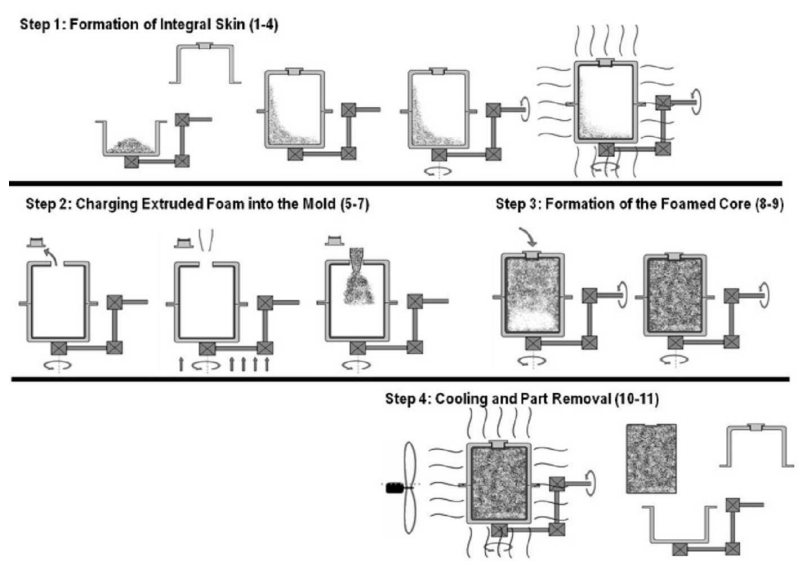

Fig. 2. The four processing steps of the RRFM technology.

switched to a uni-axial mode and the mold aligned with the extrusion port. The extruder subsequently fills the hot mold with extrudate comprised of the desired foam for a period of time dependent on the melt flow rate and screw RPM. The filling process occurs at the moldextruder interface gate, which has been designed so as to seal the mold during the conventional rotational molding cycle, facilitate the introduction of foam to it during the foam filling stage, and allow the skin to self-heal after the filling process is accomplished thereby allowing the skin to settle back at its original location, undamaged.

Step 4: The mold is then bi-axially rotated and cooled. Subsequently, the solidified part is removed.

\section{Experimental}

A dedicated industrial-grade, custom-made, heavyduty, lab-scale, semi-automatic, experimental setup has been successfully designed, developed, and built (Fig. 1). This apparatus was used as a test bed for producing extrusion-assisted cellular morphologies and evaluating 

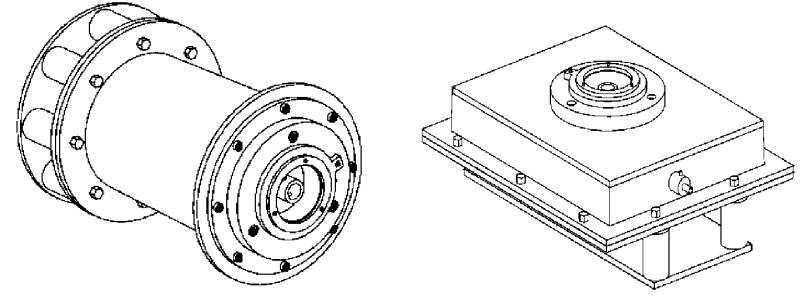

Fig. 3. The two mold shapes used for experimentation.

their quality as well as the accomplished savings in processing time and energy. The experimental results revealed that the transfer of this breakthrough technology would provide a new class of quality rotational moldings at substantially lower energy and production costs, which will advance rotational foam processors to the forefront of the international competition.

Typical Properties of Microthene® MP643662

\begin{tabular}{l|c|c|c|c}
\hline \hline Property & Nominal value & Units & Test method & Sample \\
\hline Melt Index & 3.6 & $\mathrm{~g} / 10 \mathrm{~min}$. & ASTM D 1238 & Pellets \\
Density & 0.9395 & $\mathrm{~g} / \mathrm{cc}$ & ASTM D 1505 & Compression molded \\
Tensile Strength, Yield, 2" /min & 2,600 & $\mathrm{psi}$ & ASTM D 638 & Rotomolded \\
Heat Distortion Temp. @ 66 psi & 52 & ${ }^{\circ} \mathrm{C}$ & ASTM D 648 & Rotomolded \\
@ 264 psi* & 40 & ${ }^{\circ} \mathrm{C}$ & ASTM D 648 & Rotomolded \\
Low Temp. Impact: & & & ARM STD & \\
$1 / 8$ " specimen & 51 & ft-lbs. & $\left(-40^{\circ} \mathrm{F}\right.$ impact $)$ & Rotomolded \\
$1 / 4$ " specimen & 140 & ft-lbs. & $\left(-40^{\circ} \mathrm{F}\right.$ impact $)$ & Rotomolded
\end{tabular}

Typical Properties of Microthene® MP652762

TABLE II

\begin{tabular}{l|c|c|c|c}
\hline \hline Property & Nominal value & Units & Test method & Sample \\
\hline Melt Index & 2.0 & $\mathrm{~g} / 10 \mathrm{~min}$. & ASTM D 1238 & Pellets \\
Density & 0.942 & $\mathrm{~g} / \mathrm{cc}$ & ASTM D 1505 & Compression molded \\
Tensile strength field, 2" /min & 3,220 & $\mathrm{psi}$ & ASTM D 638 & Rotomolded \\
Heat Distortion Temp. @ 66 psi & 67 & ${ }^{\circ} \mathrm{C}$ & ASTM D 648 & Rotomolded \\
@ 264 psi* & 42 & ${ }^{\circ} \mathrm{C}$ & ASTM D 648 & Rotomolded \\
Low Temp. Impact: & & & ARM STD & \\
1/8" specimen & 80 & ft-lbs. & $\left(-40^{\circ}\right.$ F impact $)$ & Rotomolded \\
1/4" specimen & 190 & ft-lbs. & $\left(-40^{\circ}\right.$ F impact $)$ & Rotomolded
\end{tabular}

The HDPE (Microthene@) MP652762) was UVstabilized and had a melting point of $128.31^{\circ} \mathrm{C}$ and a crystallization temperature of $117.14^{\circ} \mathrm{C}$. Table II presents the typical properties of Microthene $\mathbb{R}$ MP652762 according to manufacturer's data.

\subsection{Design of experimental molds}

Two mold shapes made of Aluminum 2014 have been used for experimentation, a "cylindrical" and a "flat panel" mold as presented in Fig. 3 .

The cylindrical mold had a base radius of $11 \mathrm{~cm}$ and a total height of $17 \mathrm{~cm}$ which set its total volume to be $\sim 1400 \mathrm{~cm}^{3}$. A 20 degree draft angle was incorporated in the mold design to facilitate part removal. The flat panel mold size was $23 \times 18 \times 3.5 \mathrm{~cm}$ (length $\times$ width $\times$ depth) respectively, which set its total volume to be $\sim 1450 \mathrm{~cm}^{3}$. This mold also had a 20 degrees draft angle to facilitate part removal.

\subsection{Polyethylene (PE) grades and respective $C B A$}

An LMDPE and an HDPE grade were the experimental materials tested. Both materials were made by Equistar Chemicals. The LMDPE (Microthene®) MP643662) was UV-stabilized and had a melting point of $127.23^{\circ} \mathrm{C}$ and a crystallization temperature of $115.11^{\circ} \mathrm{C}$. Table I presents the typical properties of Microthene $\mathbb{R}$ MP643662 according to manufacturer's data.

TABLE I 


\subsection{Polypropylene (PP) grades and respective $C B A$}

Four different PP resin grades were obtained from Lyondell Basell for conducting experimental trials. These $\mathrm{PP}$ grades were carefully selected to reflect a wide variety of characteristics, from systematic differences in MFIs to a selected variation in material nature from PP homopolymers to PP copolymers. The resins, received in pellet form, were initially pulverized at Ingenia Polymers to mesh 35 powders to test their processability as solid skin constituents in Rapid Rotational Foam Molding, and when used for foaming purposes, to enhance and facilitate their dispersion and distribution characteristics with the CBA. The CBA used to formulate the foamable PP resins was Celogen AZ-120 (exothermic) and was obtained from Chrompton Chemicals. The molecular constituents of this thermally activated CBA are azodicarbonamide with amorphous silica. Celogen AZ-120 was selected after comparing its performance and base properties with Celogen OT and Celogen AZ-130, this is due to the compatibility of its pertaining decomposition temperature range with the obtained $\mathrm{PP}$ grades as well as its small particle size, which assists the dispersion and distribution properties during the implemented dry blending procedure.

\section{Results and discussion}

Multiple batteries of planned Rapid Rotational Foam Molding experimental trials resulting in both polyethylene (PE)- and polypropylene (PP)-based integral-skin rotational moldings were conducted utilizing the custombuilt industrial-grade lab-scale Rapid Rotational Foam Molding experimental setup depicted in Fig. 1 and by utilizing two distinct mold shapes depicted in Fig. 3.

\subsection{PE based experimental results}

Typical optimized resulting core foam-only scanned micrographs, accompanying respective skin-foam interface micrographs are presented in Fig. 4. They show that an excellent interface between the skin and foam has been achieved, which is very desirable. Typically, LLDPE moldings with a VER of 3 had average cell sizes

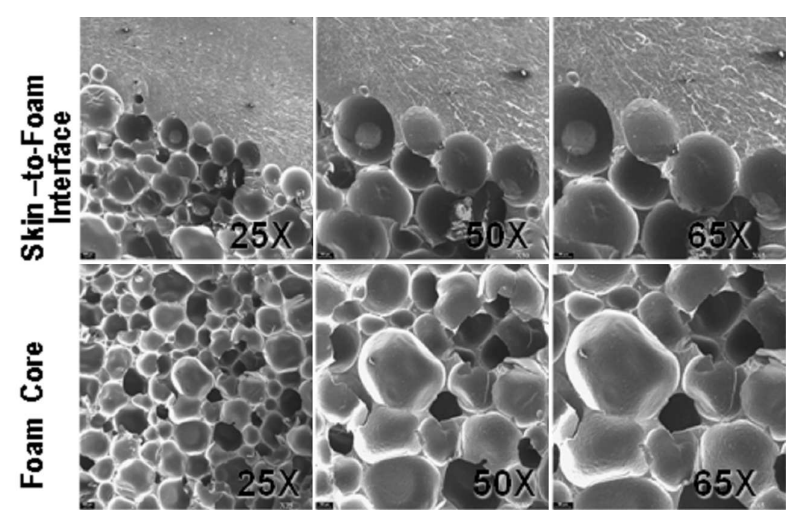

Fig. 4. PE skin / PE foam SEM morphologies.

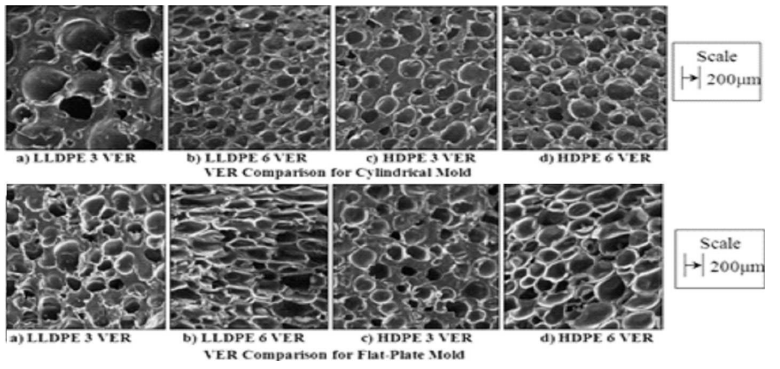

Fig. 5. Effect of VER, PP grade and mold shape on foam morphology.

larger than for the moldings with a 6 VER. However, visible in both moldings with a VER of 3 was that there were larger gaps between cells, resulting in increased cell to cell distances that would increase the average cell size and reduce the cell population density. This could be caused by the reduction in the amount of CBA used in the foamable formulation. Micrographs of both resins at the varying VER values created with both the cylindrical and the flat-plate mold, as presented in Fig. 5, were analyzed. Again, increased cell to cell distances were observed resulting in increased average cell sizes and reduced cell population densities for the moldings with a 3 VER.

\subsection{PP based experimental results}

Typical optimized resulting core foam-only SEM micrographs, accompanying respective skin-foam interface micrographs are presented in Fig. 6. The SEM micrographs revealed a strong bond between the distinct solid skin and the foamed core. This is justified by the fact that in Rapid Rotational Foam Molding the foaming extrudate is introduced within the skin boundaries at the time when the skin is still in a semi-molten/soft state in the hot uni-axially rotating mold, which allows for an excellent adherence of both layers together as the cooling process commences. This feature is extremely advantageous and was not achievable before.

In total, three distinct PP-based materials formulations, of different morphological, compositional, and rheological natures, were investigated throughout the concluding experimental work. The thoroughly analyzed results proved the success of the Rapid Rotational Foam Molding concept in processing integral-skin foamed core PP moldings for all three formulations. In terms of integral-skin quality, a bubble-free non-degraded solid integral skin was achieved all along the accomplished experimental series with calculated variability in skin thickness uniformity as low as $8.9 \%$ and as high as $33.7 \%$.

Two PP homopolymers, PFHL451H (MFI = $2 \mathrm{~g} / 10$ min) and PF6523 (MFI $=4 \mathrm{~g} / 10 \mathrm{~min}$ ), with the second sustaining a MFI that is twice as high as the first, were proved to achieve successful moldings through the experimental runs. The achieved average cell densities, when utilizing both resins for foaming purposes, were rather 


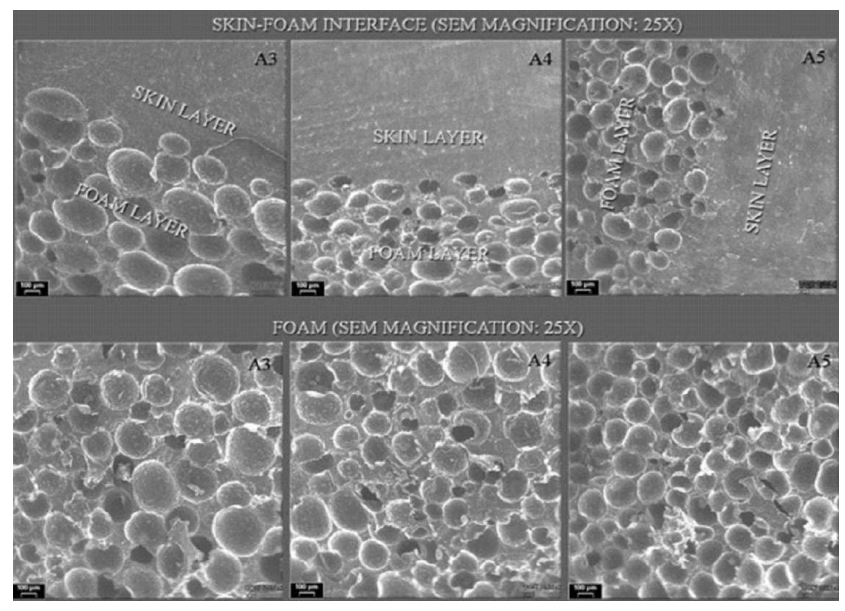

Fig. 6. PP skin / PP foam SEM morphologies.
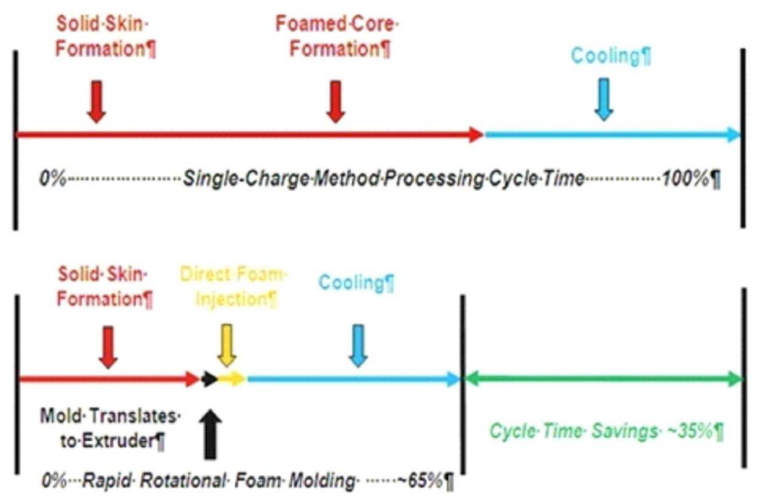

Fig. 7. Cycle duration comparison: single charge method vs. RRFM method.

comparable, with PF6523 exhibiting a slightly higher average foam density when foamed with respect to both, the 4 -fold and a 6 -fold formulation. The distribution of the foam structure associated with PFHL451H appeared to be more uniform than that of PF6523.

Also, the lowest average cell size calculated for the foam obtained using PFHL451H proved to be smaller than the one obtained for PF65234, particularly at the higher VER. This result allowed concluding that the PP grade with the lower MFI preserved its melt strength over the one with higher MFI during foaming in Rapid Rotational Foam Molding. This contradicts to previous research conclusions about foaming PP in rotational foam molding, however this can be explained by the fact that in Rapid Rotational Foam Molding the foaming was performed in extrusion rather than in a long time rotating mold in an oven, which appears to be another important advantage of the Rapid Rotational Foam Molding process in comparison to the common single charge process. Compared to conventionally-processed respective analogs, the presented experimental results revealed that the Rapid Rotational Foam Molding process has been successfully validated while offering a $35 \%$ less time consuming (Fig. 7) and a less energy intensive processing method in comparison with common single charge rotational foam molding method. A future research and development goal includes exploring the feasibility of processing microcellular rotationally molded foams by modifying the newly developed processing concept so that it could even work by using a physical blowing agent (PBA) such as $\mathrm{CO}_{2}$ [21-37].

\section{Conclusion}

Rapid Rotational Foam Molding completely decuples the part's solid skin shaping and the formation of the skin-encapsulated foamed layer or core thereby improving process controllability and shortening the cycle duration for approximately $35 \%$, which translates into a noteworthy energy consumption reduction. The experimentally obtained foam morphologies and skin-foam interfaces were satisfactory for both PE and PP grades. As melt extrusion has been successfully integrated into the rotational foam molding process, it advantageously becomes possible to eventually implement physical blowing agents in rotational foam molding for producing a novel class of microcellular polyolefin composites which will possess properties that are currently not achievable.

\section{References}

$>$ REFERENCES UNCHANGED $<\quad$ SHOULD BE REVISED ACCORDING TO APPA RULES http://www.ifpan.edu.pl/APP/guide.pdf

1. G.L. Beall, Rotational Molding, Hanser/Gardner, Cincinnati, 1998.

2. J.L. Throne, Thermoplastic Foams, Sherwood Techn., Inc., Sherwood Publ., Ohio, 1996.

3. R.J. Crawford, Rotational Moulding of Plastics, $2^{\text {nd }}$ Ed., John Wiley and Sons Inc., 1996.

4. R.J. Crawford, Plastics Engineering, Third Edition, Butterworth Heinemann, 1998.

5. G. Liu, C.B. Park, and J.A. Lefas, Polym. Eng. Sci., Vol. 38, pp. 1997, 1998.

6. S.-J. Liu and C.-H. Yang, SPE, ANTEC, Technical Papers, 46, pp. 1309, 2000.

7. R. Pop-Iliev, G.M. Rizvi and C.B. Park, Polym. Eng. Sci., Vol. 43, No. 1, pp. 40-54, 2003.

8. R. Pop-Iliev, C.B. Park, and S. D'Uva, SPE RETEC, Independence, Ohio, pp. 85, 2002.

9. M.P. Kearns and R.J. Crawford, Rapra Technology Ltd., Heidelberg, Paper 12, pp. 123, 2002.

10. S.-J. Liu and C.-H. Tsai, Polym. Eng. Sci., Vol. 39, pp. 1776, 1999.

11. S.-J. Liu and C.-H. Yang, Adv. Polym. Tech., 20, pp. 108, 2001.

12. Equistar Chemicals Company, Equistar Rotomolding Tech. Topics \#2-\#5, 2001.

13. J. Throne, SPE ANTEC, Technical Papers, 46, pp. 1304, 2000. 
14. J. Throne, SPE RETEC, Technical Papers, Independence, Ohio, pp. 79, 2002.

15. E. Takacs, J. Vlachopoulos, and S. Lipsteuer, SPE, RETEC, Technical Papers, pp. 15, 1999.

16. R. Pop-Iliev, G. Liu, F. Liu, C.B. Park, S. D'Uva, and J.A. Lefas, SPE RETEC, pp. 95, 1999.

17. R. Pop-Iliev, F. Liu, G. Liu and C.B. Park, Adv. Pol. Techn., Vol. 22, 4, pp. 280-296, 2003.

18. C.B. Park, G. Liu, F. Liu, R. Pop-Iliev, S. D'Uva, B. Zhang, U.S. Pat. 6,103,153, 2000.

19. R. Pop-Iliev and C.B. Park, J. of Reinf. Pl. and Comp., Vol. 21, No. 2, pp. 101-120, 2002.

20. R. Pop-Iliev, N. Dong, D. Xu, and C.B. Park, Adv. Pol. Techn., 2008, 26/4, 213.

21. R. Pop-Iliev, K-H Lee, and C.B. Park, J. of Cell. Plastics, 2006, 42/2, 139 .

22. D. Xu, R. Pop-Iliev, C.B. Park, R. Fenton, J. of Cell. Plastics, 2005, 41/6, 519

23. R. Pop-Iliev, D. Xu and C.B. Park, J. of Cell. Plastics, 2004, 40/1, 13.

24. R. Pop-Iliev and C.B. Park, J. of Cell. Plastics, 2003, $39 / 1,49$
25. R. Pop-Iliev, K. Christian and E. Abdalla in PPS 25 Proceedings, Goa, 2008, CD

26. E. Abdalla and R. Pop-Iliev in SPE ANTEC Proceedings, Chicago, 2009, CD.

27. K. Christian, E. Abdalla, G. Eberle, G., and R. PopIliev in SPE ANTEC, Chicago, 2009, CD.

28. R. Pop-Iliev in PPS 24 Proceedings, Keynote Presentation, Salerno, 2008, CD.

29. K. Christian and R. Pop-Iliev in SPE Foams Proceedings, Charlotte, 2008, CD.

30. E. Abdalla, K. Christian and R. Pop-Iliev in SPE ANTEC Proceedings, Milwaukee, 2008, CD.

31. R. Pop-Iliev in SPE ANTEC Proceedings, Cincinnati, 2007, CD.

32. K. Christian, MASc Thesis, UOIT-University of Ontario Institute of Technology, 2009.

33. E. Abdalla, MASc Thesis, UOIT-University of Ontario Institute of Technology, 2009.

34. R. Pop-Iliev in SPE ANTEC Proceedings, Orlando, 2010 CD. 